\title{
Carbon-13 CP MAS NMR Study on Structures of Octadecyl Chains Influenced by Co-Presence of 3-Aminopropyl Chains on SBA-15
}

\author{
Oc Hee Han, Yoon Kyung Bae, and Soon Yong Jeong \\ Analysis Research Division, Daegu Center, Korea Basic Science Institute, Daegu 702-701, Korea. ${ }^{*}$ E-mail: ohhanakbsi.re.kr \\ Received November 8, 2007
}

\begin{abstract}
Functionalized SAB-15 samples by octadecyltrimethoxysilane (OTC) were studied by ${ }^{13} \mathrm{C}$ magic angle spinning (MAS) cross polarization (CP) nuclear magnetic resonance (NMR) spectroscopy. In the SBA-15 sample fully functionalized by 3-aminopropỵltrimethoxysilane (APS) and OTC in 1:1 molar ratio. octadecyl chains were observed to have. on average. more trans conformation than those in the SBA-15 samples fully modified by OTC only. Our results confirm that long chain molecules tend to organize themselves better in the co-presence of short chain molecules on the surface of mesoporous materials by packing of the different length chains in an interdigitized fashion even when the short chains are long enough to have three carbons and a functional group at the ends. In addition. our results indicate that solid-state ${ }^{13} \mathrm{C} C \mathrm{CPAS}$ NMR spectroscopy is a simple and non-destnictive method to probe the molecular structures of the domains composed of long alkỵl chains.
\end{abstract}

Key Words : Solid-state NMR spectroscopy, SBA-15, 3-Aminopropyltrimethoxysilane, Octadecyltrimethoxysilane. Functionalized mesoporous materials

\section{Introduction}

SBA-15 is a highly ordered mesoporous silica material in a two-dimensional hexagonal (space group $p 6 \mathrm{~mm}$ ) structure and typically produced in acidic media with a block copolymer. ${ }^{1}$ The pore size (up to $-30 \mathrm{~nm}$ ) and the thichness of the silica wall can be tunable with variable block copolymers and SBA-15 structures have thicker silica wall than MCM-4l structures. ${ }^{1}$ Surface functionalized mesoporous silicate materials has been regarded to have the great potential for applications in environmental and industrial processes such as adsorption, separation, and catalysis. Not only the functional groups but also molecular strictures of the surface bound species of the functionalized mesoporous materials play an important role in efficient utilization of the materials. Due to the chemical shifts sensitive to conformations as well as configurations and the NMR signal related with molecular dynamics. solid-state NMR spectroscopy has been entoloyed as one of the main analytical methods to probe the molecular structures and dynamics of the surface bound ${ }^{3,5}$ and intercalated organic species. ${ }^{0,7}$ Especially. the structures and dynamics of long alkyl chains have been studied intensively by solid-state NMR spectroscopy ${ }^{--8}$ In this work, ${ }^{13} \mathrm{C}$ CP MAS NMR is demonstrated as a simple and quick method to check the molecular structure of long alkyl chains bound on the surface. The SAB-15 samples. fully functionalized with OTC only, APS only, and OTC and APS together in 1:1 molar ratio. are compared.

\section{Experimental}

SBA-15 was synthesized as reported previously, with

"Present address: School of Advanced Materials Science \& Engineering. Sungkyunkwan University. Suwon 440-746. Korea triblock poly(ethylene oxide)-poly(propylene oxide)-poly(ethylene oxide) $\left(\mathrm{EO}_{20}-\mathrm{PO}_{70}-\mathrm{EO}_{20}\right.$. PI23: Aldrich. U.S.A.) copolymer as a structure directing agent and tetraethyl orthosilicate (TEOS; Aldrich, U.S.A.) as a silica source. In a typical synthesis, $4 \mathrm{~g}(0.69 \mathrm{mmol})$ of PI23 was dissolved in $30 \mathrm{~g}$ of water and $120 \mathrm{~g}$ of $2 \mathrm{M} \mathrm{HCl}$ solution and then stirred at $35^{\circ} \mathrm{C}$ for an hour. Then. $8.5 \mathrm{~g}(40.8 \mathrm{mmol})$ of TEOS was added dropwise into the homogeneous solution with stirring at $35^{\circ} \mathrm{C}$ for $20 \mathrm{~h}$. The obtained gel was aged at $100^{\circ} \mathrm{C}$ without stirring for $24 \mathrm{~h}$. The solid white product was filtered. washed with distilled water and air-dried at room temperature. The dried SBA-15 sample was heated in a tube fumace under air by slowly increasing temperature at $1{ }^{\circ} \mathrm{C} /$ min from room temperature to $550^{\circ} \mathrm{C}$ and then calcined at the temperature for 6 hours. Then the furnace was tumed off to cool naturally under air or dry nitrogen atmosphere. The small angle X-ray Diffraction (XRD) pattern for the calcined SBA- 15 was consistent with the XRD patterns in reference 1. indicating our calcined SBA-15 is in $p 6 \mathrm{~mm}$ hexagonal symmetry. However. our diffraction peaks appear at slightly smaller $2 \theta$ values corresponding to larger unit cell parameters and the intense ( 100$)$ peak reflects a $d$ spacing of $-10.2 \mathrm{~lm}$.

Prior to functionalization reactions with OTC and/or APS, SBA-15 materials were refluxed in toluene for 24 hours to increase the hydroxyl group density ${ }^{15}$ on the surface of SBA-15. Appropriate amount of APS and/or OTC was injected to $2 \mathrm{~mL}$ of absolute toluene under dry nitrogen gas flow. The refluxed and filtered SBA-15 (200 mg) was slowly added to the toluene solution for $10 \mathrm{~min}$ with vigorous stirring. Then the mixture was refluxed at $100^{\circ} \mathrm{C}$ with stirring for 24 hours. The solid white product was filtered and washed with absolute toluene and dichloromethane.

All solid-state NMR spectra were acquired on a DSX-400 

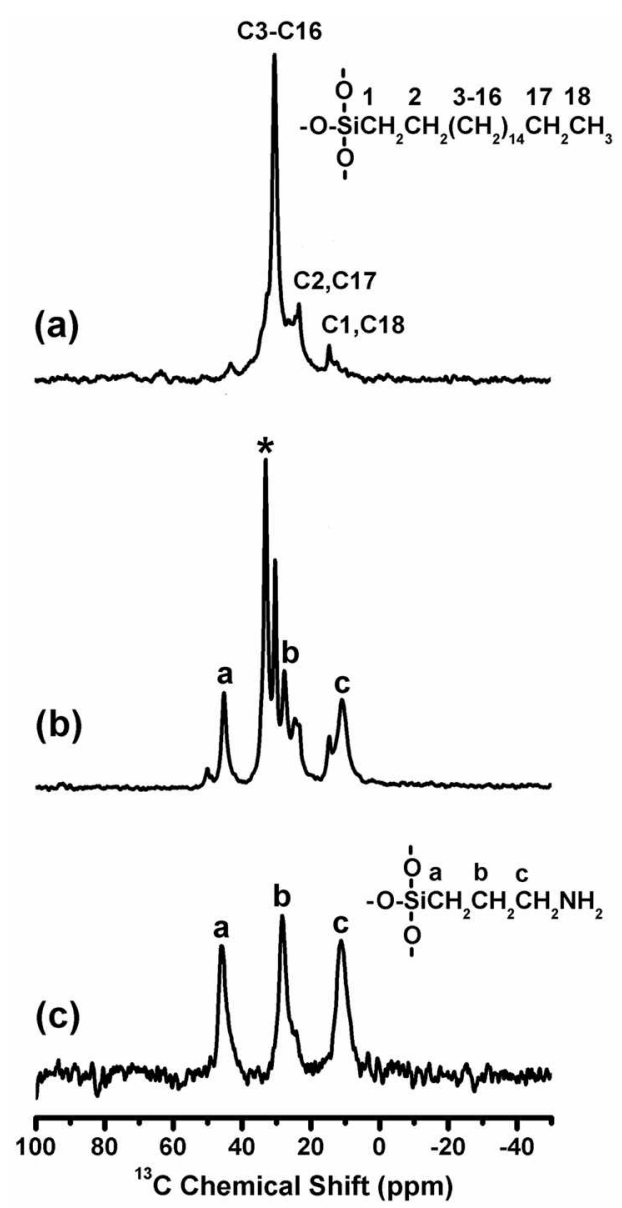

Figure 1. Representative ${ }^{13} \mathrm{C}$ CP MAS NMR spectra of SBA-15 functionalized with OTC only (a), OTC and APS in 1:1 molar ratio (b), and APS only (c). The peak at 30 ppm in (a) and (b) is assigned to the $\mathrm{C} 3-\mathrm{Cl} 6$ in trans-ganche misture confonmation while the additional peak at $33 \mathrm{ppm}$ in (b) (marked by $\star$ ) is assigned to the C3-C 16 in all trans conformation. The peaks not marked in (b) exactly match with the peaks in (a). Refer to the main test for additional information on the peak assignments.

spectrometer (Bruker Biospin GmbH. Germany) with a magnetic field strength of $9.4 \mathrm{~T}$ and $4 \mathrm{~mm}$ rotors at room temperature. ${ }^{13} \mathrm{C}$ CP MAS spectra were acquired with $3 \mathrm{~s}$ pulse sequence repetition delay. $5 \mu$ s proton pulse length ( $90^{\circ}$ flip), $2 \mathrm{~ms}$ contact time, a spectral width of $100 \mathrm{kHz}$. and a spinning rate of $6 \mathrm{kHz}$. All chenical shifts in ppm were referenced to extemal tetramethylsilane.

\section{Results and Discussion}

Representative ${ }^{13} \mathrm{C}$ CP MAS NMR spectra of the SBA-15 samples functionalized with OTC only, OTC and APS in $1: 1$ molar ratio. and APS only in a saturation coverage are shown in Figure la. 1b. and lc respectively. The peaks in Figures la and $1 c$ were assigned as denoted by comparison to the spectra simulated with the ChemDraw program and consistent with the previous reports...11 It can be easily recognized that the peaks of 3-aminopropyl chains attached on SBA-15 did not change their chemical shifts even in the co-presence of octadecyl chains on the surface of SBA-15.
On the other hand. a new peak from the internal carbons (C3-CI6) of octadecyl chains appears at $33 \mathrm{ppm}$ in the copresence of 3-aminopropyl chains on the surface of SBA-15 in addition to the peak at $30 \mathrm{ppm}$ for the $\mathrm{C} 3-\mathrm{Cl} 16$ in the absence of 3-aminopropyl chains.

It is quite well-known that ${ }^{13} \mathrm{C}$ chemical shifts of the methylene carbons located in the middle of a long alkyl chain are very sensitive to their conformation. ${ }^{5-7}$ The methylene carbons in all trans conformation have a chemical shift between 34.2 and $32.8 \mathrm{ppm}$ according to their molecular packing and motional state while the chemical shift of those in trans-gatche mixture conformation is upfield shifted. ${ }^{3.57}$ The more gauche conformation is present. the more chemical shift is upfield shifted. The maximum shift of methylene carbons due to conformation difference was reported as $-5 \mathrm{ppm}{ }^{12}$ Hence. the chemical shift of the C3CI6 peak. at $30 \mathrm{ppm}$. for octadecyl chains on the SBA-15 surface functionalized only with OTC indicates that the carbons are in trans-gatche mixture confonmation. The additional peak at $33 \mathrm{ppm}$ in Figure $\mathrm{lb}$ suggests a new functionalized surface domain in which the $\mathrm{C} 3-\mathrm{Cl} 6$ carbons are in an all trans conformation. Hence. by co-codensation with APS the new domain was produced in addition to the domain functionalized with octadecyl only chains with the C3-C16 in trans-gatche mixture conformation as observed in the SBA-15 sample functionalized OTC only. Our results indicate that OTC was not homogeneously co-condensed with APS on the surface of SBA-15 and APS co-condensed with OTC induced all trans conformation for the $\mathrm{C} 3-\mathrm{Cl} 6$ of the octadecyl chains next to 3-aminopropyl groups. As a result, the population of trans conformation of the octadecyl chains was, on average. increased in the presence of 3aminopropyl groups on the surface of SBA-15. Previously it was reported that the mixture of molecules with a linear propyl chain capped with a non-carbon functional group (a long chain molecule) and a methyl carbon only (a short chain molecule) for surface modification produces denser surface functionalization and better hydrothermal stability than either molecules only. ${ }^{11.13}$ Our results imply that even when the short chain is longer than a methyl group. the mixture of surface functionalization molecules with a long chain and a short chain produces better ordered long chain molecules on the surface. However, more than a single peak for the $\mathrm{C} 3-\mathrm{C} 16$ of the octadecyl chains show that the surface functionalization with the mixture of OTC and APS were done inhomogeneously and resulted in two different surface domains or phases: the one functionalized with OTC only and the other functionalized with 3-aminopropyl and octadecyl groups together on the SBA-15 surface. Outer surface of SBA-15 is also functionalized during our functionalization process. however. the contribution of NMR signal from the functionalized outer surface is negligible compared to that from the functionalized inner surface. Therefore. the detection of all trans conformation suggests the pore size of the SBA-15 is large enough to hold this extended octadecyl chains. Although the better ordering manifested as all trans conformation can be explained by interdigitation of short 
chain molecules and long chain molecules on the silica surface. the explanation does not have enough supporting data and the mechanism for the better ordering is not clear at this moment. In general, ${ }^{13} \mathrm{C}$ CP MAS spectra can be acquired in much shorter time than ${ }^{13} \mathrm{C}$ MAS spectra without $\mathrm{CP}$. However, peak intensities of ${ }^{13} \mathrm{C}$ CP MAS spectra tend to intensify less mobile carbons with more protons nearby and consequently are not linearly proportional to the number of carbons related with each peak.

\section{Conclusion}

Our results demonstrate that ${ }^{13} \mathrm{C}$ CP MAS NMR spectroscopy can provide a lot of information on molecular structures even with a simple experiment. especially on the conformation of alkyl chains and surface functionalization domains. Systematic studies will be pursued in the near future to search the optimum condition for homogeneous functionalization of mesoprorous materials and the relationship between the structures and dynamics of the octadecyl chains and the molar ratios of APS and OTC in the cocondensation. How the better ordering of the long alkyl chains is produced in the presence of short chain molecules will be probed. In addition, ${ }^{13} \mathrm{C}$ CP MAS and ${ }^{13} \mathrm{C}$ MAS without CP NMR data will be compared to develop a method to get quantitative infornation from ${ }^{13} \mathrm{C}$ CP MAS NMR spectra.

Acknowledgements. This work is supported by the Korea
Research Foundation Grant. KRF-2003-015-C00410. Prof. Jong-Sung Yu at Hannam University and Ms. S. H. Kim at KBSI are acknowledged for teclunical assistance at our initial stage of SBA-15 preparation and in NMR experiments, respectively.

\section{References}

1. Zhao. D.: Feng. T.: Huo. Q.: Melosh. N.: Fredrickson. G. H.: Chmelka. B. F.: Stuchy. G. D. Science 1998. 279. 548.

2. Liu. T.: Feng. X.: Fryxell. G. E.: Wang. L. Q.: Kimn. A. Y.: Gong. M. Adv Aater 1998. 10, 161.

3. Liu. J.; Shin, Y.: Nie. Z.: Chang, J. H.: Wang, L.-Q.: Fryell, G. E; Samuels, W. D; Exarhos. G. J. J. Plys. Chem. A 2000. 104. 8328.

4. Bae. J. Y.: Choi. S.-H.: Bae. B.-S. Bull Korem Chem. Soc. 2006. 27. 1562 .

5. Gao. W: Reven, L. Langmir 1995, 11. 1860.

6. Wang, L.-Q; Liu. J.; Exarhos, G. J.: Flanigan. K. Y; Bordia, R. J. Phus. Chem. B 2000. 10+, 2810 and references therein.

7. Han1. O. H.: Paik. Y.: Moon. Y. S.: Lee. S. K.: Kim. T. Y.: Lee. Y. H.: Lee. W. I. Chent Mater 2007. 19.3615.

8. Ishikawa. S.: Kuroso. H.: Ando. I. J. Mol. Sintct. 1991. 2+8.361 and references therein.

9. Zhao. D; Huo, Q; Feng. J.: Chmelka, B. F; Stucky, G. D. I. Am Chem. Soc. 1998, 120.6024.

10. Luechinger. M.: Pritns. R.: Pirngruber. G. D. Micropor. Mesopor. Mater 2005. 85.111 and references therein.

11. Maria Chong. A. S.: Zhao. X. S. J. Phls Chem. B 2003. 107. 12650 .

12. Tonelli, A. E:; Schilling. F. C. Acc. Chem. Res. 1981. 14. 233.

13. Yang. H.: Zhang, G.; Hong. X.: Zhu. Y. Mifropor Mesopor Mater: 2004. 68. 119 . 